\title{
Alterações fonoaudiológicas presentes em um caso de síndrome de Goldenhar
}

\section{Language and hearing characterization in the Goldenhar syndrome: case report}

\author{
Fernanda Thieme Lima ${ }^{1}$, Camila Brenner de Araújo ${ }^{2}$, Elaine Colombo Sousa ${ }^{3}$, Brasília Maria Chiari ${ }^{4}$
}

\begin{abstract}
RESUMO
A linguagem é um complexo processo que envolve a participação e a interação de todos os seus componentes: fonologia, semântica, morfologia, sintaxe e pragmática. A aquisição e organização desses componentes estão relacionadas a capacidades internas do ser humano e também com seu ambiente. Qualquer comprometimento em um ou mais desses componentes trará conseqüências para o desenvolvimento da linguagem de maneira geral. Na Síndrome de Goldenhar diferentes comprometimentos podem afetar a capacidade do indivíduo de se comunicar. Sendo assim, o objetivo deste estudo foi descrever as alterações fonoaudiológicas encontradas em uma criança portadora de Síndrome de Goldenhar e fornecer informações adicionais que possam contribuir para adequação da intervenção fonoaudiológica. A criança, do sexo feminino, 8 anos e 8 meses de idade, foi submetida à avaliação da linguagem, fala, voz, audição e motricidade oral. Foi observada alteração dos aspectos cognitivos da linguagem: noção de orientação espacial, raciocínio lógico abstrato e memória e atenção; bem como alteração da expressão e compreensão oral, de órgãos fonoarticulatórios, das funções neurovegetativas, e da audição (perda auditiva bilateral). Inúmeros casos da Síndrome de Goldenhar foram descritos na literatura. No entanto, a grande maioria destes dá ênfase à descrição das alterações genéticas e fenotípicas presentes nos indivíduos estudados. Assim, os achados fonoaudiológicos do presente estudo não podem ser generalizados para todos os portadores da Síndrome de Goldenhar, sendo este o relato de um único caso. Tais achados evidenciam a necessidade da atuação diagnóstica e interventiva do fonoaudiólogo em equipe multidisciplinar, nos quadros de síndromes genéticas.
\end{abstract}

Descritores: Síndrome de Goldenhar/diagnóstico; Transtornos da comunicação; Transtornos do desenvolvimento da linguagem; Transtornos da audição; Assimetria facial

\section{INTRODUÇÃO}

Comunicação, do latim communicatio, pode ser definida como um meio pelo qual o indivíduo relata experiências,

(1) Especializanda em Linguagem pelo Curso de Especialização em Fonoaudiologia da Faculdade de Medicina da Universidade de São Paulo FMUSP - São Paulo (SP), Brasil.

(2) Fonoaudióloga clínica; Especialista em Distúrbios da Comunicação Humana pelo Departamento de Fonoaudiologia da Universidade Federal de São Paulo - UNIFESP - São Paulo (SP), Brasil.

(3) Especializanda em Distúrbios da Comunicação Humana pelo Departamento de Fonoaudiologia da Universidade Federal de São Paulo UNIFESP - São Paulo (SP), Brasil.

(4) Professora Titular do Curso de Fonoaudiologia da Universidade Federal de São Paulo - UNIFESP - São Paulo (SP), Brasil; Coordenadora da PósGraduação em Distúrbios da Comunicação Humana Stricto-Senso do Departamento de Fonoaudiologia da Universidade Federal de São Paulo UNIFESP - São Paulo (SP), Brasil.

Trabalho desenvolvido no Ambulatório de Avaliação e Diagnóstico Fonoaudiológico da Universidade Federal de São Paulo - UNIFESP - São Paulo (SP), Brasil.

Endereço para correspondência: Fernanda Thieme Lima. R. Abaúna, 508, São Paulo - SP, CEP 04284-080. E-mail: fernanda_thiemelima@yahoo.com.br Recebido em: 9/11/2006; Aceito em: 2/2/2007 idéias, conhecimentos e sentimentos ao outro. A mensagem a ser compartilhada é codificada na fonte e decodificada no destino, com o uso de sistemas de signos ou símbolos sonoros, iconográficos ou gestuais, entre outros ${ }^{(1)}$.

A linguagem é um desses sistemas e constitui-se em um processo complexo que envolve a participação e a interação de todos os seus componentes: fonologia, semântica, morfologia, sintaxe e pragmática. A aquisição e organização desses componentes estão relacionadas a capacidades internas do ser humano, como a maturação do sistema nervoso central $^{(2)}$, e também com seu ambiente, que deve ser rico em estímulos e possibilitar diversas experiências lingüísticas.

Há diversas formas de distúrbios de comunicação que podem afetar a capacidade de ouvir e de entender o que os outros dizem. Podem, ainda, comprometer a voz, a fluência e articulação dos sons da fala, os símbolos da linguagem e o código utilizado na comunicação ${ }^{(3)}$.

Diferentes comprometimentos encontrados na síndrome de Goldenhar podem afetar a capacidade do indivíduo de se comunicar.

A síndrome de Goldenhar, primeiramente descrita em 
1952 por Maurice Goldenhar, é uma anomalia congênita rara, caracterizada por alterações oculares, auriculares e vertebrais, freqüentemente associadas a outras malformações congênitas viscerais ou faciais ${ }^{(4)}$. Acredita-se que a síndrome de Goldenhar faça parte de um quadro clínico mais complexo de anomalias de primeiro e segundo arcos branquiais, o qual é mais comumente conhecido como espectro óculo-aurículovertebral e é caracterizado pela presença adicional de dermóides epibulbares ${ }^{(5)}$.

A maioria dos casos da referida síndrome é esporádica ${ }^{(5-6)}$, mas já foram relatados casos familiares com herança autossômica dominante com expressividade variável e, também, casos em que existe consangüinidade entre os pais, sugerindo herança autossômica recessiva ${ }^{(6)}$.

Sua etiologia ainda é desconhecida. As causas mais comumente relatadas são: anormalidades cromossômicas, distúrbio na migração das células neurais, diabetes materna, fatores ambientais durante a gravidez e ingestão de drogas pela mãe, tais como: cocaína, talidomida, ácido retinóico e tamoxifen $^{(4)}$.

Tal espectro varia significativamente em padrão e severidade. Sua incidência varia em uma faixa de 1 para 5600 recém-nascidos. A relação entre meninos e meninas portadores da síndrome é de aproximadamente 3:2. A freqüência em relação aos lados afetados (direito versus esquerdo) é de $1: 1^{(6)}$.

$\mathrm{Na}$ literatura, são descritas como características da síndrome a presença de microssomia hemifacial, hipoplasia mandibular, malformações auriculares (protuberâncias ou fístulas pré-auriculares), dermóides epibulbares, manifestações ortopédicas, anormalidades esqueléticas e alterações oculares. Também está associada à microtia ipsilateral, hidrocefalia e retardo mental; além de anormalidades sistêmicas: cardiovasculares, pulmonares, labirínticas, traqueoesofágicas, renais e genitourinárias ${ }^{(4-7)}$.

Inúmeros casos de síndrome de Goldenhar foram descritos na literatura. No entanto, a grande maioria destes enfatiza a descrição das alterações genéticas e fenotípicas presentes, havendo uma escassez de achados em relação às habilidades comunicativas dos indivíduos estudados. Assim, o presente estudo tem como objetivo descrever as características fonoaudiológicas observadas em uma criança portadora da síndrome de Goldenhar.

\section{APRESENTAÇÃo DO CASO CLÍNICO}

O presente estudo foi aprovado pelo Comitê de Ética da Universidade Federal de São Paulo - Escola Paulista de Medicina sob o número 0980/ 06.

Trata-se de uma criança de oito anos e oito meses de idade, do sexo feminino, avaliada e diagnosticada no Ambulatório de Avaliação e Diagnóstico Fonoaudiológico da Universidade Federal de São Paulo (UNIFESP) devido à queixa familiar de trocas na fala e dificuldades escolares.

Para a caracterização das manifestações fonoaudiológicas, a paciente foi submetida aos seguintes procedimentos: (a) anamnese com os pais ${ }^{(8)}$; (b) avaliação audiológica básica ${ }^{(9)}$, que constou de audiometria tonal liminar, logoaudiometria e imitanciometria; (c) avaliação da linguagem expressiva e receptiva, na qual foi aplicado o Teste $\mathrm{ABFW}^{(10)}$, nas áreas de Fonologia, Vocabulário e Pragmática para verificação dos aspectos fonético-fonológicos, de competência lexical do individuo e aspectos funcionais da comunicação, respectivamente, e a prova de complementação de sentenças do Exame de Linguagem TIPITI $^{(11)}$, que visa avaliar a estrutura e disposição das palavras nas frases do discurso oral (morfologia e sintaxe); (d) avaliação miofuncional ${ }^{(8)}$, na qual foram averiguados o aspecto facial e os órgãos fonoarticulatórios, a partir da observação da dentição, da postura de repouso, da movimentação e da palpação dos lábios, língua e bochechas. A avaliação do padrão respiratório e das funções neurovegetativas foi realizada por meio de observações da atuação dos órgãos fonoarticulatórios durante a fala, a mastigação e a deglutição de saliva, alimentos sólidos e líquidos; (e) triagem do desenvolvimento, que constou da aplicação do Teste de Triagem do Desenvolvimento DENVER II $^{(12)}$, que consistiu da avaliação de itens, divididos em grupos (pessoal-social, motor fino-adaptativo, linguagem e motor-grosseiro), sendo os itens administrados à criança ou informados pelo responsável, segundo critérios pré-estabelecidos no teste. Os itens foram então analisados e classificados como "passa", "falha", "recusa-se" ou "não houve oportunidade", de acordo com o desempenho da criança na tarefa solicitada. Embora a faixa etária da paciente ultrapasse aquela para qual o instrumento foi elaborado, optamos por sua utilização frente aos achados na avaliação psicológica realizada anteriormente.

Os testes audiológicos referidos anteriormente foram realizados em cabina acústica, utilizando-se o audiômetro MA41, da marca Maico e o imitânciometro AZ7, da marca Interacoustics.

Todos os testes aplicados foram analisados e interpretados segundo critérios de normalidade adotados na literatu$\mathrm{ra}^{(8-12)}$.

Segundo dados da anamnese, a criança é a terceira filha de pais saudáveis e não-consangüíneos. A idade materna ao nascimento era de 27 anos. A criança nasceu de parto cesáreo, pós-termo e houve ingestão de mecônio, sendo necessária uma lavagem estomacal. Por conta disso, a menina ficou em incubadora durante dois dias. Peso e estatura não foram relatados.

Alterações físicas foram notadas logo ao nascimento. A criança foi, então, encaminhada ao serviço de genética e exames pertinentes levaram ao diagnóstico de síndrome de Goldenhar.

Aos seis anos de idade, o Centro de Psicologia da APAESP diagnosticou deficiência mental de grau leve, sendo o atraso mental da criança de três anos.

À observação física, realizada aos oito anos e oito meses de idade, a criança apresentava assimetria de face, sendo o lado direito mais severamente afetado: orelha, olho e boca possuíam implantação mais elevada. Além disso, apresentava malformação do pavilhão auricular direito e microssomia.

$\mathrm{Na}$ avaliação dos órgãos fonoarticulatórios, foi verificada a presença de palato profundo, dentição mista em bom estado de conservação e assimetria do lábio inferior para a es- 
querda. Lábios e língua possuíam tônus diminuído. A mastigação era realizada bilateralmente, sem centralização do bolo alimentar e com desvio da mandíbula para o lado esquerdo. Na deglutição, havia interposição de língua, anteriorização da cabeça e resíduos alimentares no vestíbulo, após várias deglutições. A criança era respiradora oral e ficava constantemente com os lábios entreabertos. Sua voz tinha intensidade aumentada.

Quanto aos aspectos motores, estes se mostraram dentro do esperado para a faixa etária da criança.

Os exames audiológicos revelaram perda auditiva mista, de grau moderado a moderadamente severo, na orelha direita e perda auditiva neurossensorial de grau leve a moderado, na orelha esquerda.

A criança apresentava compreensão oral apenas quando fazia uso de pistas orofaciais. Sua fala tinha inteligibilidade alterada, apresentando processos fonológicos não adequados a sua idade (plosivação de fricativas, posteriorização para velar e para palatal, frontalização de velares e palatais, simplificação de líquidas e simplificação da consoante final). Apresentava vocabulário inadequado para sua idade, restrito a vocalizações e jargões ininteligíveis, associados a gestos indicativos e representativos, além de alteração morfossintática na estrutura frasal devido a ininteligibilidade de sua fala.

Quanto aos aspectos cognitivos da linguagem, a criança apresentava noção de corpo e capacidade de discriminar cores, formas e tamanhos. No entanto, apresentava dificuldades em nomear as formas. Foi observada a presença de funcionalidade e jogo simbólico.

A criança apresentava alterações quanto à noção de orientação espacial, não fazia relação cognitiva com quantidade e não realizava categorizações. Também possuía dificuldades em raciocínio lógico abstrato e dificuldades de memória e atenção.

Os resultados obtidos com a avaliação fonoaudiológica foram discutidos e chegou-se a hipótese diagnóstica sindrômica de Distúrbio de Aquisição e Desenvolvimento de Fala e Linguagem ${ }^{(8)}$.

\section{DISCUSSÃO}

A síndrome de Goldenhar é um espectro de anormalidades craniofaciais, vertebrais e sistêmicas. O estudo desta condição ainda é controverso devido à sua complexidade e abrangência de aspectos clínicos.

A maioria dos casos de síndrome de Goldenhar é esporádica, mas já foram relatados casos familiares com herança autossômica dominante com expressividade variável, e também casos em que existe consangüinidade entre os pais, sugerindo herança autossômica recessiva ${ }^{(6)}$. No presente caso, os pais da criança não eram consangüíneos e a história familiar era negativa, estando em concordância com a maioria dos casos já relatados na literatura ${ }^{(4-5)}$.

A presença de retardo mental em portadores da síndrome de Goldenhar é bastante discutida entre os pesquisadores. A paciente em estudo apresentou deficiência e atraso mental, concordando com os achados de alguns autores ${ }^{(5-6)}$. Contu- do, a grande maioria dos estudiosos não faz menção a este tipo de alteração.

É importante salientar que um aspecto considerado como pré-requisito básico para a aquisição da linguagem é o desenvolvimento intelectual. Segundo a literatura, um dos critérios básicos para o diagnóstico de retardo mental é a função intelectual significativamente abaixo da média, demonstrada por um quociente de inteligência (QI) igual ou menor que 70. Vários autores pesquisaram e concluíram que existe uma relação direta entre o aparecimento da fala e o QI rebai$\mathrm{xado}^{(13)}$. Assim, as alterações de fala e linguagem relatadas no presente estudo não podem ser interpretadas, unicamente, como decorrentes da síndrome, devendo estar associadas ao retardo mental, apresentado pela paciente.

Até o presente momento, não há concordância sobre os critérios mínimos para o diagnóstico da síndrome de Goldenhar; contudo, a maioria dos autores sugere a presença de microssomia como a alteração mais comumente obser$\operatorname{vada}^{(4-5,7)}$. A criança em estudo apresentou assimetria de face e mal-formações de pavilhão auricular. Tais características são citadas em diferentes estudos e caracterizam a $\operatorname{microssomia}^{(4-7)}$.

Assim, as alterações das funções neurovegetativas encontradas na criança podem ser justificadas pela microssomia e assimetria de face.

Com relação à voz, a literatura descreve ausência de distúrbios vocais orgânicos ou funcionais na síndrome de Goldenhar $^{(14)}$, diferente do que foi observado no caso objeto deste estudo que apresentou intensidade de voz aumentada.

Diferentemente dos achados vocais, os achados audiológicos estão em acordo com os relatados na literatura( ${ }^{(7)}$. Entretanto, o indivíduo em estudo apresentou perda auditiva bilateral, sendo que a maioria dos casos descritos apresenta audição bilateral normal ${ }^{(4,7)}$, perda auditiva condutiva, mista ou neurossensorial unilateral ${ }^{(5,7)}$.

É preciso enfatizar que a deficiência auditiva apresentada pela paciente indica riscos para alteração de linguagem, uma vez que a integridade do sistema auditivo é fundamental para o desenvolvimento da linguagem. Segundo descrito na literatura, alterações auditivas acarretam alterações de linguagem, pois juntamente à maturação auditiva ocorre o desenvolvimento de fala e linguagem ${ }^{(15)}$.

As alterações na compreensão e expressão de linguagem foram descritas por Van Lierde et al. ${ }^{(14)}$. Em um de seus estudos, tais autores descreveram alterações na linguagem expressiva relacionadas à morfossintaxe, alterações de vocabulário receptivo e dificuldades para compreensão oral de sentenças ${ }^{(14)}$. A compreensão oral da criança em estudo era eficaz apenas com o uso de pistas orofaciais. Além disto, esta apresentava vocabulário restrito e estrutura frasal alterada, concordando com os achados descritos anteriormente.

$\mathrm{O}$ mesmo estudo ${ }^{(14)}$ descreveu as alterações encontradas na articulação de crianças com síndrome de Goldenhar. Tais crianças foram capazes de produzir todas as vogais e consoantes de sua língua materna, e os erros apresentados por elas foram, principalmente, ensurdecimento, distorções e omissão da consoante final, do encontro consonantal, da sílaba átona e da consoante inicial. A criança estudada apresentou 
inteligibilidade de fala alterada, com a presença de processos fonológicos não adequados para a idade. Além de apresentar simplificação da consoante final, como descrita na literatura, apresentou plosivação, posteriorização e frontalização de fonemas.

As alterações sobre os aspectos cognitivos da linguagem estão de acordo com as informações que aparecem comentadas na literatura, embora sem detalhamento ${ }^{(7)}$.

\section{COMENTÁRIOS FINAIS}

Muitos são os casos de síndrome de Goldenhar descritos na literatura. No entanto, a grande maioria destes dá ênfase à descrição das alterações genéticas e fenotípicas presentes nos indivíduos estudados. As informações sobre as característi- cas de linguagem e audição aqui mencionadas podem ser de grande auxílio para o entendimento desses indivíduos. Entretanto, os achados fonoaudiológicos descritos não podem ser generalizados para todos os portadores da síndrome de Goldenhar, sendo este o relato de um único caso.

Considerando as manifestações gerais e específicas/ fonoaudiológicas, que interferem nas habilidades de comunicação desta síndrome, evidenciadas neste estudo, é importante ressaltar a atuação diagnóstica e interventiva do fonoaudiólogo em equipe multidisciplinar, nos quadros de síndromes genéticas.

Cabe ressaltar, que o diagnóstico e intervenção fonoaudiológica, quando realizados precocemente, fornecem a criança melhores oportunidades de desenvolvimento lingüístico.

\begin{abstract}
Language is a complex process that involves the participation and interaction of all of its components: phonology, semantics, morphology, syntax and pragmatics. The acquisition and organization of these components are related to internal capacities of the human being and also to his environment. Any implication in one or more of these components brings consequences to language development in general. In Goldenhar's syndrome, different implications may affect the individual's ability to communicate. Thus, the purpose of this research was to describe the speech, language and hearing impairments found in a child with Goldenhar's syndrome and to provide additional information that might contribute to the apropriate speech-language and hearing intervention. The child, a female 8 years and 8 months old, was submitted to language, speech, voice, hearing and oral motricity evaluations. Deficits in the cognitive aspects of language were observed: spatial orientation notion, logical abstract reasoning, memory and attention; as well as deficits of oral expression and comprehension, phonoarticulatory organs, neurovegetative functions and hearing (bilateral hearing loss). Many cases of Goldenhar's syndrome have been described in literature. However, great part of them emphasize the description of genetic and fenotipic manifestations of the studied individuals. Hence, the speech-language and hearing findings of the present study cannot be generalized to all of the individuals with Goldenhar's syndrome, since this is a report of single case. These findings evidence the need for the diagnostic and interventive participation of the speech-language and hearing therapist as part of a multidisciplinary team, in cases of genetic syndromes.
\end{abstract}

Keywords: Goldenhar syndrome/diagnosis; Communication disorders; Language development disorders; Hearing disorders; Facial asymmetry

\title{
REFERÊNCIAS
}

1. Nicolosi L, Harryman E, Kresheck J. Vocabulário dos distúrbios da comunicação: fala, linguagem e audição. 3a ed. Porto Alegre: Artes Médicas; 1996.

2. Vitto MMP, Feres MCLC. Distúrbios da Comunicação Oral em Crianças. Medicina (Ribeirão Preto). 2005;38(3/4):229-34.

3. Boone DR, Plante E. Uma introdução à comunicação humana e seus distúrbios. In: Boone DR, Plante E. Comunicação humana e seus distúrbios. 2a ed. Porto Alegre: Artes Médicas; 1994. p.9-15.

4. Pinheiro AL, Araujo LC, Oliveira SB, Sampaio MC, Freitas AC. Goldenhar's syndrome - case report. Braz Dent J. 2003;14(1):67-70.

5. La Barca Lleonart M, Paz Sarduy A, Ocaña Gil MA, Atienza Lois L. Displasia oculoauriculovertebral o síndrome de Goldenhar. Estudio multidisciplinario de un caso clínico. Rev Cuba Oftalmol. 2001;14(1):42-6.

6. Tasse C, Böhringer S, Fisher S, Lüdecke HJ, Albrecht B, Horn D, et al. Oculo-auriculo-vertebral spectrum (OAVS): clinical evaluation and severity scoring of 53 patients and proposal for a new classification. Eur J Med Genet. 2005;48(4):397-411.
7. Brosco KC, Zorzetto NL, Costa AR. Perfil audiológico de indivíduos portadores da síndrome de Goldenhar. Rev Bras Otorrinolaringol. 2004;70(5):645-9.

8. Perissinoto J, Chiari BM. A avaliação como ação precursora do diagnóstico. In: Andrade CRF, Marcondes E, coordenadores. Fonoaudiologia em pediatria. São Paulo: Sarvier; 2003. p.135-40.

9. Munhoz MSL, Silva MLG. A avaliação audiológica no exame otoneurológico. In: Ganança MM, Vieira RM, Caovilla HH, editores. Princípios de otoneurologia. São Paulo: Atheneu; 1998. v.1. p. 7-21. [Série Distúrbios de Comunicação Humana].

10. Andrade CRF, Befi-Lopes DM, Fernandes FDM, Wertzner HF. ABFW Teste de Linguagem Infantil nas áreas de fonologia, vocabulário, fluência e pragmática. 2a ed. Carapicuíba: Pró-Fono; 2004.

11. Braz HA, Pellicciotti THF. Exame de linguagem TIPITI. São Paulo: MNJ; 1988.

12. Frankenburg WK, Dodds J, Archer P, Shapiro H, Bresnick B. The Denver II: a major revision and restandardization of the Denver Developmental Screening Test. Pediatrics. 1992;89(1):91-7. 
13. Silva AA. O diagnóstico nos distúrbios da comunicação. In: Marchesan IQ, Bolaffi G, Gomes ICD, Zorzi JL, organizadores. Tópicos em fonoaudiologia. 2a ed. São Paulo: Lovise; 1995. p.475-82.

14. Van Lierde KM, Van Cauwenberge P, Stevens I, Dhooge I. Language, articulation, voice and resonance characteristics in 4 children with
Goldenhar syndrome: a pilot study. Folia Phoniatr Logop. 2004;56(3):131-43.

15. Northern JL, Downs MP. Desenvolvimento do comportamento auditivo. In: Northern JL, Downs MP. Audição em crianças. 3a ed. São Paulo: Manole; 1989. p.101-41. 